\title{
Asymmetric field flow fractionation applied to the nanoparticles characterization: Study of the parameters governing the retention in the channel
}

Valentin de Carsalade du pont ${ }^{1}$, Enrica Alasonati ${ }^{1}$, Sophie Vaslin-Reimann ${ }^{1}$, Michel Martin ${ }^{2}$, Mauricio Hoyos ${ }^{2}$, and Paola Fisicaro $^{2}$

${ }^{1}$ Department of Biomedical and Inorganic Chemistry, Laboratoire National de Métrologie et d'Essai (LNE), 1 rue Gaston Boissier, Paris, 75015, France

${ }^{2}$ Laboratoire de Physique et Mécanique des Milieux Hétérogènes (PMMH), ESPCI Paris, CNRS, 10 rue Vauquelin, 75231, Paris Cedex 05, France

\begin{abstract}
In this work we study the parameters which are often modified to optimize the separation in asymmetrical field flow fractionation, and we observe their impact on the retention behavior of the analyte. The aim of the work is to improve our knowledge of the phenomena which govern the behavior of the analytes in the channel and to have a better understanding of the limits of the actual theoretical model in order to improve it. Results illustrate that the ionic strength influences the effect of the cross flow rate on the retention time of the particle. The question of the determination of the channel thickness was also addressed.
\end{abstract}

\section{Introduction}

Since some years, researchers are interested in nanoscience to understand the original properties of nano-objects, design new nanomaterials for various applications like electronics, cosmetics or medicine, or to study the fate of nanoparticles (NPs) in the environment. The fast development of nanotechnologies created the need for analytical methodologies able to characterize the nanoparticles. The size and the size distribution are key parameters of NPs which have been included in (ISO/TS 80004-2, 2015). One of the analytical methods used to determine the size distribution of nanoparticles is the field flow fractionation (FFF).

FFF is a family of techniques which belongs to the category of separation technique like chromatography. The technique consists in the application of a field force perpendicularly to a laminar elution flow rate, allowing the particles to be separated according to the nature of the force applied. The main difference between chromatography and FFF is that the separation is not based on the affinity between the analytes and the stationary phase but on the interaction between the analytes and a force applied perpendicularly to the elution flow (Figure 1). 

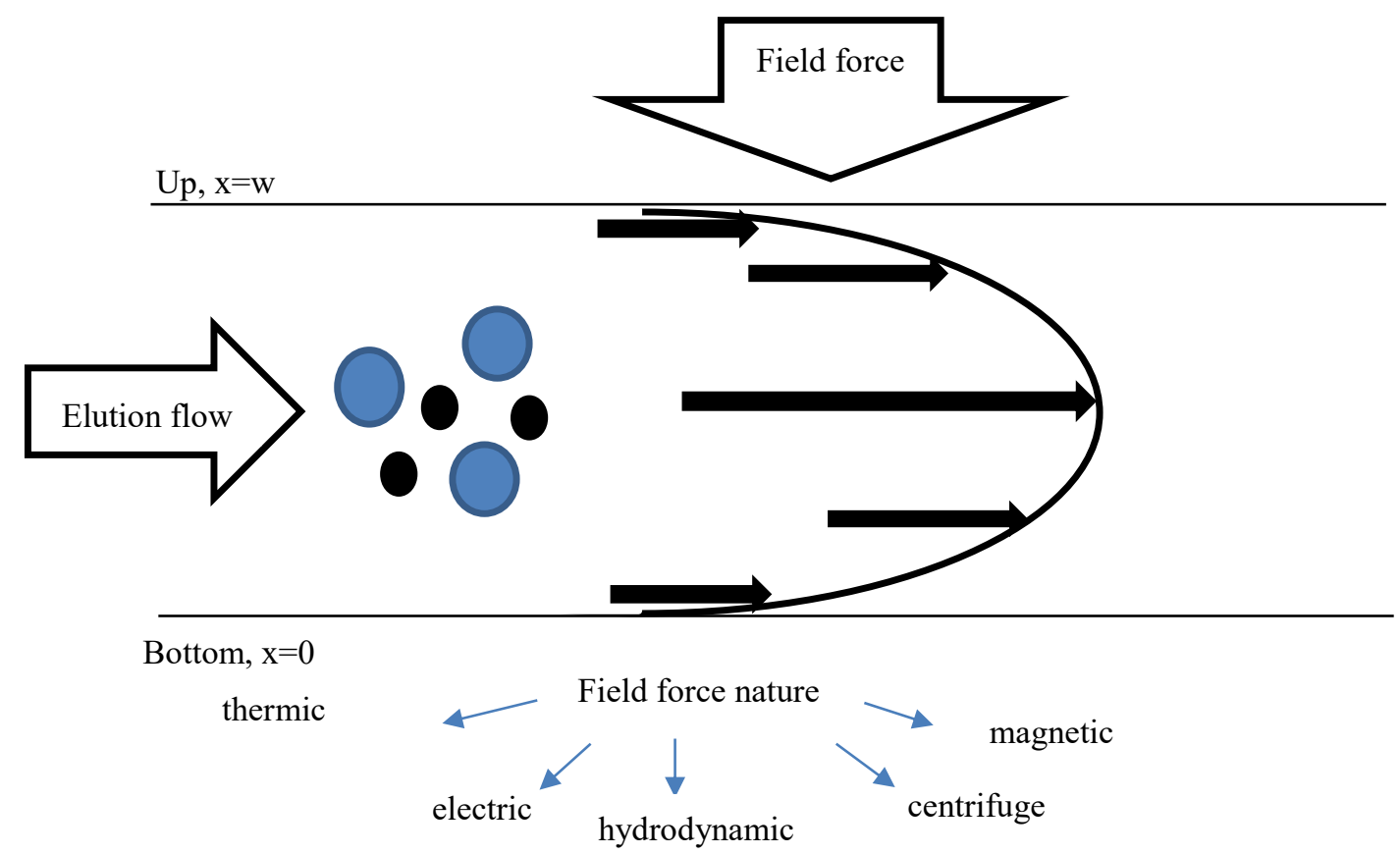

Figure 1. Separation principle of FFF

The sub techniques of FFF are distinguished by the nature of the force applied. Flow field flow fractionation (FlFFF) is a sub technique which fractionates the sample by applying a hydrodynamic force on the sample. This force is generated by a secondary flow, the cross flow, applied perpendicularly to the main one. The channel is constituted of two porous walls and a semi permeable membrane that covers one of them. Another version of the Fl-FFF was developed by Granger et al.[1] in which the channel had only one porous wall. This model has the advantage to decrease the perturbation from the cross flow generated by the heterogeneous permeability of the two porous walls. This version was called asymmetrical flow field flow fractionation (A4F)[2]. To ensure that the cross flow and the carrier velocity are constant thorough the channel, the channel was modified from a rectangular to a trapezoidal shape.

\section{2. theory}

In FFF there are different types of elution depending on the size of the analyte. In the nanoscale the elution mode followed by the nano-objects is the Brownian mode (or normal mode). A theoretical model was developed in 1960s by Giddings[3] to predict the behavior of an analyte in a FFF channel.

The measurand in FFF, like in chromatography, is the retention time $\left(t_{r}\right)$ of the eluting peak. The model defines a retention ratio $R$ which corresponds to the ratio between the velocity of the analyte zone and the velocity of the carrier. In practice it is equal to the ratio of the void peak $\left(t_{0}\right)$ and the retention time[4]:

$R=\frac{t_{0}}{t_{r}}=\frac{v_{\text {analyte }}}{v_{\text {carrier }}}$ 
Due to the large ratio width/thickness $(y / x)$ of the channel, generally larger than 60 , the channel can be assimilated to two parallel plates so that the velocity ratio can be expressed only in function of the $\mathrm{x}$ axis:

$\frac{v_{\text {analyte }}}{v_{\text {carrier }}}=\frac{\int_{0}^{w} c(x) v(x) d x}{\langle v(x)\rangle \int_{0}^{w} c(x) d x}$

where $w$ is the thickness of the channel and $v(x)$ and $c(x)$ are respectively the velocity of the flow rate and the concentration profile in the transversal axe, $x$. For the expression of $v(x)$ a laminar flow has been assumed.

$v(x)=6\langle v(x)\rangle\left(\left(\frac{x}{w}\right)-\left(\frac{x^{2}}{w^{2}}\right)\right)$

The expression of $c(x)$ has been made with the assumption of an analyte cloud in a steady state regime where only two opposite forces are applied on the analyte: the field force and the diffusion. This leads to equation (4):

$c(x)=c_{0} \exp \left(-\frac{F x}{k T}\right)$

where $c_{0}$ is the analyte concentration at the accumulation wall, $F$ is the strength applied on the analyte, $k$ the Boltzmann constant and $T$ the temperature. Equation 3 and 4 are then introduced in equation 2, which gives

$R=6 \lambda \operatorname{coth}\left(\frac{1}{2 \lambda}\right)-12 \lambda^{2}$

with $\lambda$ a dimensionless retention parameter equal to $k T / F w$. When $\lambda<0.02$ an approximation of equation 5 , accurate within $5 \%$, can be made:

$R=6 \lambda$

These conditions are easy to obtain when the analyte is retained in the channel. Therefore, most of the users use equation 6. In A4F the force applied on the channel can be approximated by:

$F_{A 4 F}=\frac{3 \pi \eta d_{h} V_{c} w}{k T V_{0}}$

With $\eta$ the carrier viscosity, $d_{h}$ the hydrodynamic diameter of the particle, $V_{c}$ the cross flow and $V_{o}$ the channel volume. Combining equation 7 and 6 we obtain an expression between the retention time and the hydrodynamic diameter of the particle.

$d_{h}=\frac{2 k T V_{o} t_{r}}{\pi V_{c} \eta t_{o} w^{2}}$

Among the hypotheses made to obtain equation 8, the hypothesis about the concentration profile implies that the interaction within the analytes and between the analytes and the accumulation wall are negligible with respect to the applied field. However, several works [5-7] showed that these interactions are not negligible which reduce the model validity. To overcome the model limitations for size determination of (nano)particles, macromolecules or proteins, nowadays FFF is coupled online with size detectors like multi angle light scattering (MALS)[8-10]. In this work we propose to investigate the main parameters usually optimized by the users, namely the carrier composition, the cross-flow rate and the channel thickness. Moreover, their influence on the retention time is 
compared to the retention time predicted by the model in order to have a better understanding of the model deviations and of possible improvements.

\section{Material and methods}

\subsection{A4F instrumentation}

Experiments were carried out in this study using an A4F system (AF2000 Postnova Analytics, Landsberg Germany) coupled to a UV detector (Shimadzu, Kyoto Japan) set at $254 \mathrm{~nm}$ The A4F channel was $27.7 \mathrm{~cm}$ long and narrowing in width from 2.0 to $0.5 \mathrm{~cm}$. A $350 \mu \mathrm{m}$ thick spacer and membrane in regenerated cellulose $(R C)$ at $10 \mathrm{kDa}$ cut-off (Postnova Analytics) were used for the experiments.

\subsection{Chemical and samples}

Polystyrene latex nanosphere (PS) size standards of 23, 60 and $100 \mathrm{~nm}$ of diameter (Thermo-Scientific) were used to study the effect of the particle size on the retention time. The samples were prepared by diluting the commercial solution in the carrier to obtain a solution at $13 \mu \mathrm{g} / \mathrm{g}$. The carrier was ultrapure water filtered through a $0.1 \mu \mathrm{m} \mathrm{RC}$ filter (Postnova Analytics). The ionic strength of the carrier was adjusted by adding sodium chloride, $\mathrm{NaCl}$ ( $\mathrm{Sigma}$ Aldrich). The injection volume was $60 \mu \mathrm{L}$.

\section{Results and discussion}

\subsection{Methodology of the study}

One particularity of the Fl-FFF is the use of a semi permeable membrane at the bottom of the channel. The role of this membrane is to keep the sample inside the channel and allows at the same time the carrier to flow out thorough the accumulation wall. Unfortunately, the membrane gradually ages with the number of injections. The phenomenon is well known [11,12] and can be detected in two different ways: following the decrease of the fractogram area, in this work the UV peak integral, or following the variation of the retention time. Most users use a size detector (as light scattering detectors) coupled to the A4F to determine the particle's size and in this case, they are not affected by the variation of the retention time between the injections. In our case the retention time is our measurand so we have included the membrane aging in our uncertainty budget to be able to compare different sets of data (Figure 2). 


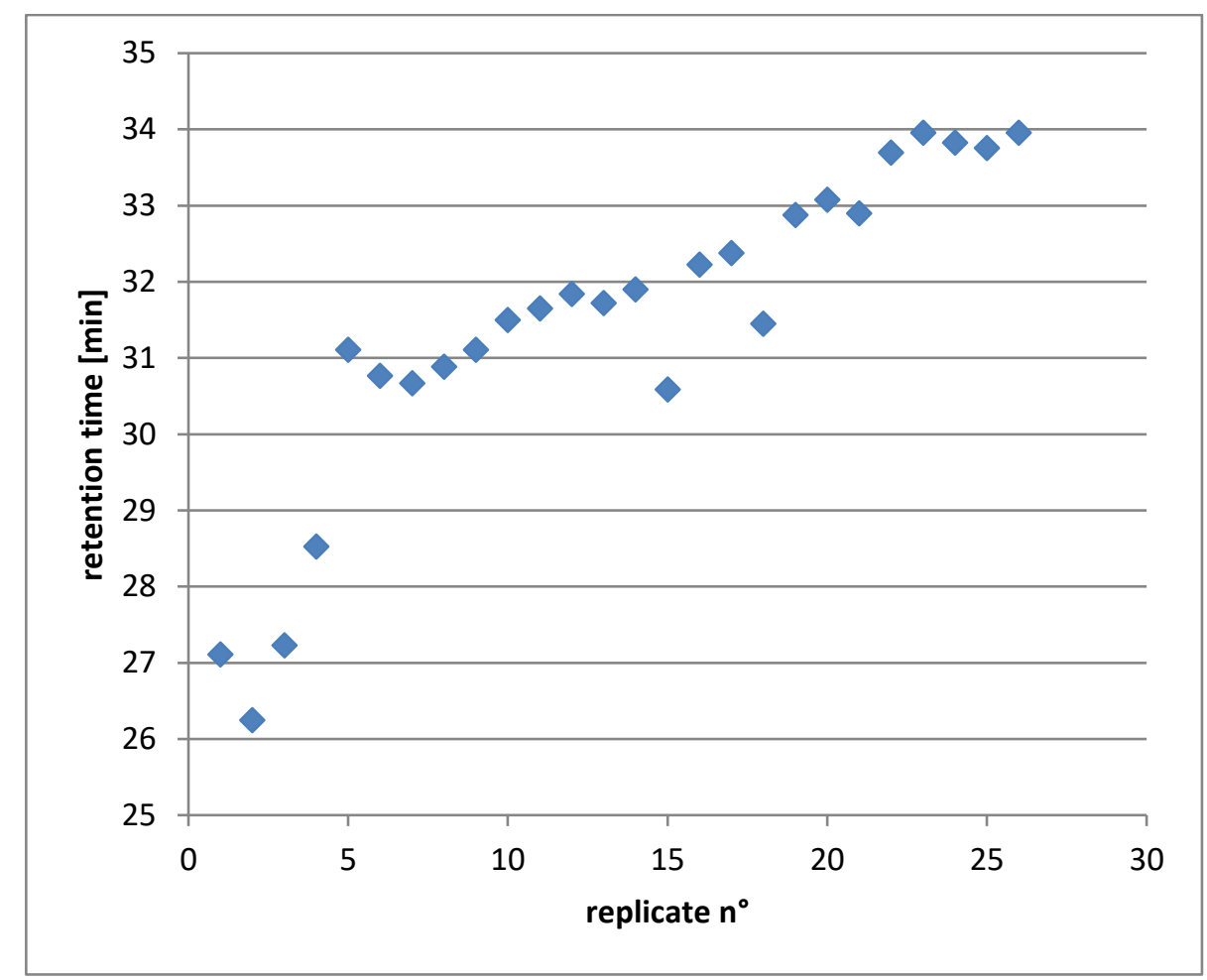

Figure 2. Retention time of the PS standard $60 \mathrm{~nm}$ as diameter in function of the number of replicates. Carrier: $\mathrm{NaCl}^{1} 10^{-}$ ${ }^{4} \mathrm{~mol} / \mathrm{L}$; membrane: $\mathrm{RC} 10 \mathrm{kDa}$; cross flow rate and elution flow rate: $0.5 \mathrm{~mL} / \mathrm{min}$.

Figure 2 represents the repeatability in $\mathrm{NaCl} 10^{-4} \mathrm{~mol} / \mathrm{L}$. If we want to test 5 five different parameters in 3 consecutive replicates, there will be 13 injections between the first and the last parameter tested which could create a significant bias larger than the repeatability of three replicates. To take the aging into account in the uncertainty budget, one series of parameters was tested first (example three different cross flow, $V_{c l}, V_{c 2}, V_{c 3}$ ), followed by 2 replicates of the same series (Table 1). This experiment plan increases the uncertainty associated to the retention time but allows the comparison between two set of data acquired at different ages of the membrane. 
Table 1. Experiment planning for three different values of cross flow $(V c)$ with 3 replicates

\begin{tabular}{|c|c|c|c|}
\hline Analysis $n^{\circ}$ & $\begin{array}{c}\text { sequence usually } \\
\text { applied }\end{array}$ & & $\begin{array}{c}\text { sequence applied in } \\
\text { this study }\end{array}$ \\
\hline 1 & replicate $1 V_{c l}$ & \multirow{9}{*}{$=>$} & replicate $1 V_{c l}$ \\
\hline 2 & replicate $2 V_{c l}$ & & replicate $1 V_{c 2}$ \\
\hline 3 & replicate $3 V_{c l}$ & & replicate $1 V_{c 3}$ \\
\hline 4 & replicate $1 V_{c 2}$ & & replicate $2 V_{c l}$ \\
\hline 5 & replicate $2 V_{c 2}$ & & replicate $2 V_{c 2}$ \\
\hline 6 & replicate $3 V_{c 2}$ & & replicate $2 V_{c 3}$ \\
\hline 7 & replicate $1 V_{c 3}$ & & replicate $3 V_{c l}$ \\
\hline 8 & replicate $2 V_{c 3}$ & & replicate $3 V_{c 2}$ \\
\hline 9 & replicate $3 V_{c 3}$ & & replicate $3 V_{c 3}$ \\
\hline $\begin{array}{c}\text { standard deviation } \\
\text { obtained }\end{array}$ & $u\left(t_{r}\right) \approx 0.4 \mathrm{~min}$ & $<$ & $u\left(t_{r}\right) \approx 2.6 \mathrm{~min}$ \\
\hline
\end{tabular}

\subsection{Impact of the ionic strength and cross flow on the retention time}

The equation 8 is based on the assumption that the interactions among analytes and between analytes and the membrane are negligible compared to the cross flow force. But this implies that the model can be only used with particular experimental conditions and, in practice, a carrier optimized for a good fractionation isn't necessarily adapted for the model (Figure 3). In the Figure 3 we show the influence of the carrier ionic strength $(I)$ on a $60 \mathrm{~nm}$ standard retention time. The retention time was measured for different cross flow rates. We can see that, depending on the carrier ionic strength, the retention time can triple. This increase limits the application of the model. Independently of the ionic strength effect on retention time, the ionic strength of the carrier also changes the effect of the cross flow on the retention. We can see that the slope of the curve change depending on the carrier ionic strength whiles it should be the same. According to Kato et al., the slope difference can be explained by a diminution of the electrostatic repulsions between the membrane and the particles due to the diminution of the length of Debye[13]. 


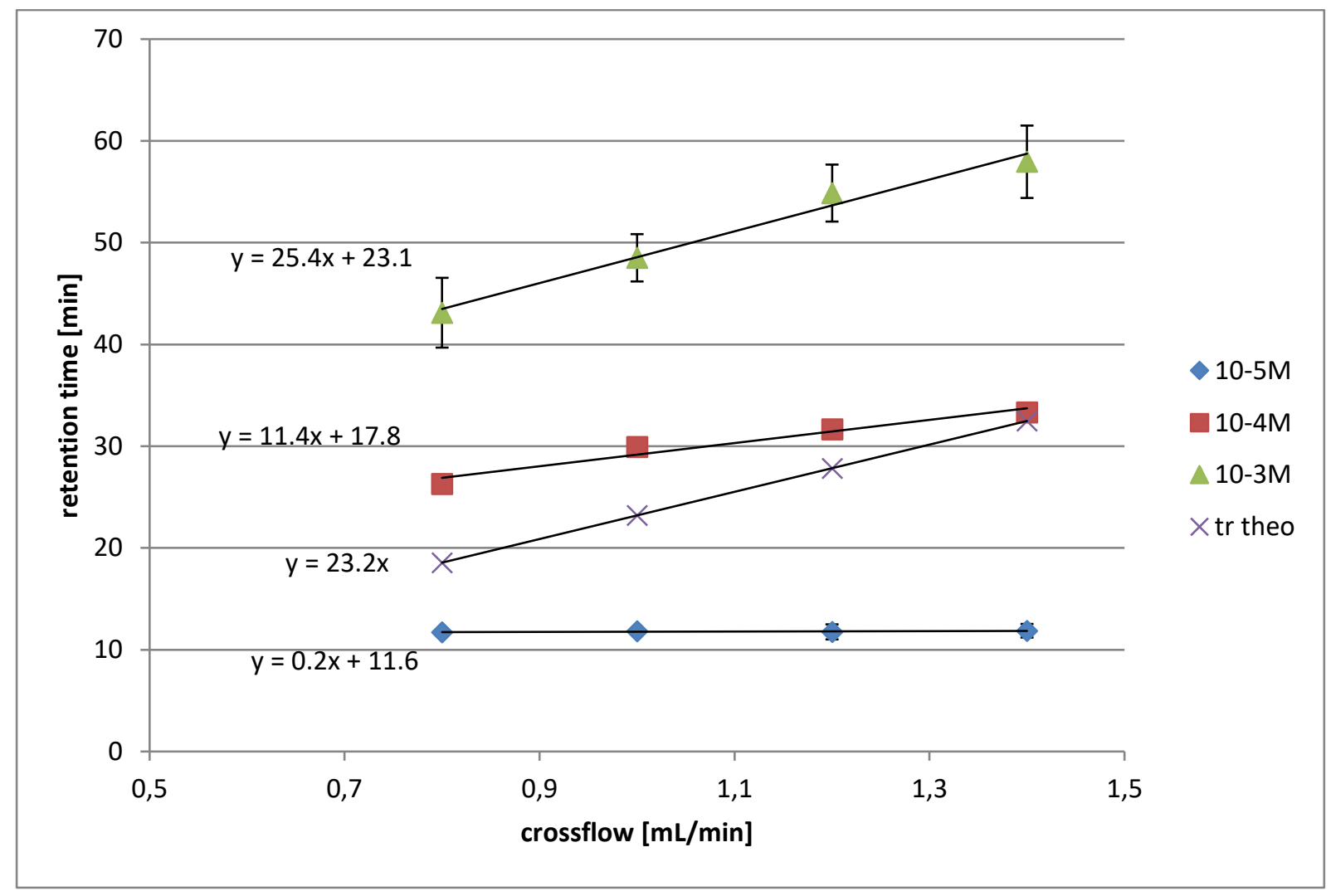

Figure 3. Retention time of PS60 in function of the cross flow for a given ionic strength. The experiment have been realized with a $\mathrm{V}_{\text {out }}=0.5 \mathrm{~mL} / \mathrm{min}$

To show the difference, the retention time predicted by the model $\left(t_{\text {rtheo }}\right)$ for a $60 \mathrm{~nm}$ particle was added in Figure 3. The parameters used for the calculation are listed in Table 2

Table 2. Value of the parameters used for the equation 8

\begin{tabular}{|cccccc|}
\hline$d_{h}[\mathrm{~m}]$ & $V o\left[\mathrm{~m}^{3}\right]$ & $\eta\left[\mathrm{kg} \mathrm{m}^{-1} \mathrm{~s}^{-1}\right]$ & $T[\mathrm{~K}]$ & $t_{0}[\mathrm{~min}]$ & $w[\mathrm{~m}]$ \\
\hline $6.00 \mathrm{E}-08$ & $1.15 \mathrm{E}-06$ & $1.00 \mathrm{E}-03$ & 293 & 1.4 & $3.50 \mathrm{E}-04$ \\
\hline
\end{tabular}

$V_{o}, t_{o}, d_{h}$ and $T$ were measured and the viscosity of the carrier was assumed equal to the viscosity of water at $293 \mathrm{~K}$. The channel thickness was assumed equal to the nominal spacer thickness even if these two values may be different in the experimental reality. This can explain the differences between the theoretic and experimental values. The theoretical curve could fit with experimental curves having the same slope, like e.g. the curve at $I=10^{-3} \mathrm{~mol} / \mathrm{L}$. if these thicknesses were equal. This inequality leads to the second principal limit in the model, the channel thickness measurement

\subsection{Channel thickness measurement}

The second principal limit of the model is the determination of the channel thickness. Indeed, when a spacer is placed in the channel, the spacer nominal thickness $\left(w_{n o m}\right)$ is known. However, two phenomena are suspected to reduce the channel thickness, each one linked with the membrane below the spacer. The first hypothesis is that the 
membrane slightly invades the spacer because of the pressure applied on the membrane when the channel is built[14]. The second supposes that, in presence of the carrier the membrane swells which also reduces the channel thickness[15]. To determine the effective channel thickness $\left(w_{\text {eff }}\right)$ a calibration is done based on equation 8 using particles or protein standards with a known hydrodynamic diameter or diffusion coefficient[16]. To evaluate the effect of the cross flow and the ionic strength of the carrier on the effective channel thickness calibration, $w_{\text {eff }}$ was calculated using a polystyrene standard of $60 \mathrm{~nm}$ as diameter by using the retention time obtained in Figure 3 (Figure 4). Each couple of conditions (cross flow and ionic strength) apart for $\mathrm{I}=10^{-5} \mathrm{~mol} / \mathrm{L}$, give a different $w_{\text {eff }}$. The equation 8 doesn't take interactions into account, hence the $w_{\text {eff }}$ were expected to vary depending on the carrier ionic strength. The cross-flow rate effect is taken into account in the calibration (equation 8) so the calculated thickness shouldn't vary with the used cross flow. However, we saw that the ionic strength also influences the effect of the cross flow rate on the particles retention time (Figure 3). So, the fact that $w_{\text {eff }}$ varies depending on the cross flow applied is not surprising (Figure 4).

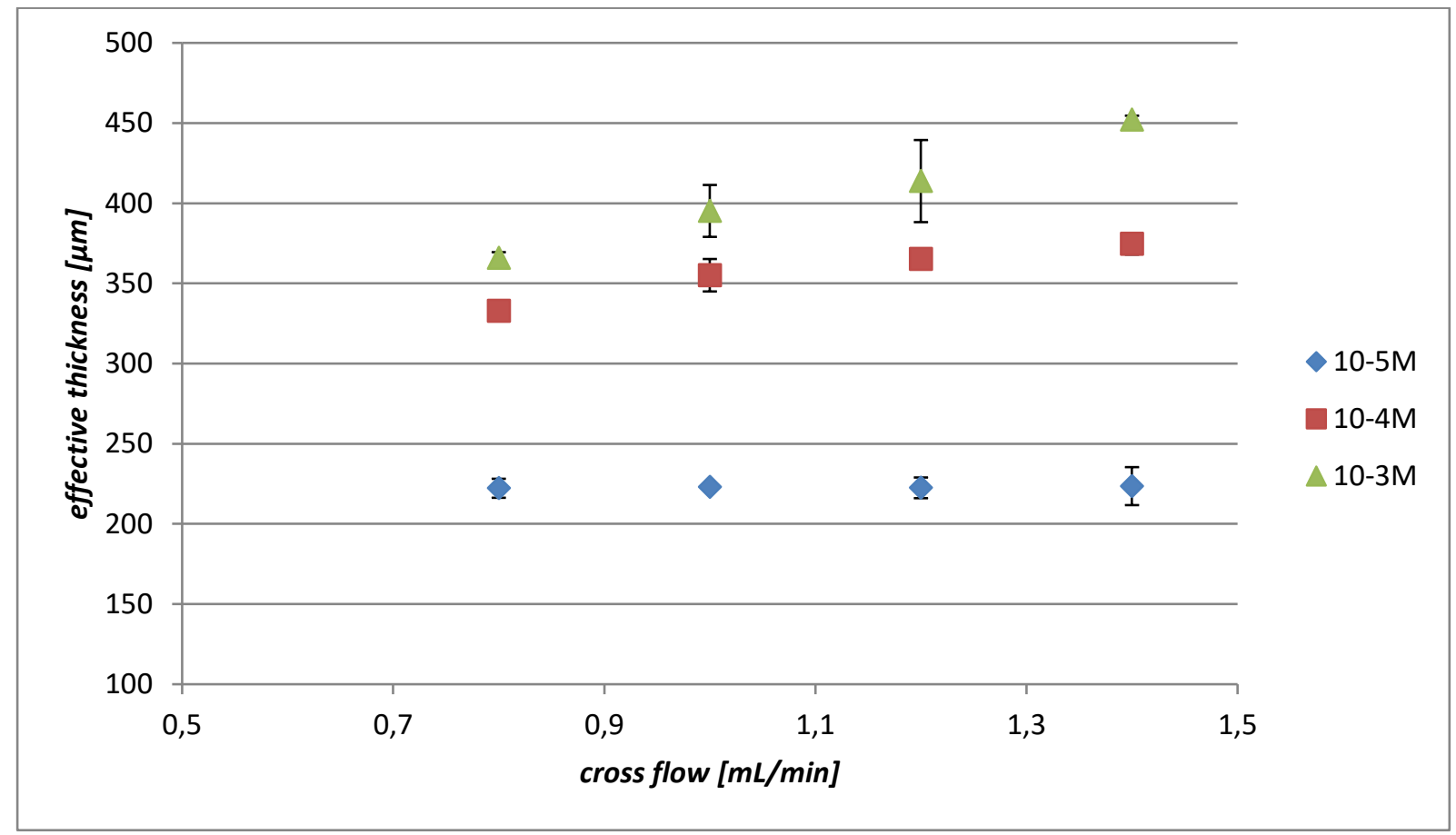

Figure 4. Determination of the effective channel thickness by using a PS standard of $60 \mathrm{~nm}$ diameter. The calibration has been performed using different carrier concentrations and different cross flow rates. The nominal channel thickness was $350 \mu \mathrm{m}$.

Depending on the experimental conditions we obtained a $w_{\text {eff }}$ which can go from $250 \mu \mathrm{m}$ to $450 \mu \mathrm{m}$. The fact that we obtain a larger or smaller $w_{\text {eff }}$ with a significant increase or decrease compared to $w_{\text {nom }}$ makes us think about the physical meaning of the $w_{\text {eff }}$ value. The two hypotheses cited above can explain the thickness decrease but not the increase. Moreover $w_{\text {eff }}$ increased proportionally with the ionic strength and the cross flow rate applied during the calibration. It could be said that the calibration approach used to determine the effective thickness of the channel results in fact in a determination of a kind of correction constant taking in account particles and membrane interactions in the model, more than an evaluation of the channel thickness.

\section{Conclusion}


In this work we have identified, experimentally measured and discussed two mains problems of the model representing the particle retention in A4F: the impact of the ionic strength and the measurement of the effective channel thickness. Ideally, particle-particle and particle-membrane interactions should be included in the forces applied on the particle: this should allow representing the different retention time tendency observed in function of the ionic strength.

The challenge due to the thickness can be solved in multiple ways. One possibility could be to assume the nonknowledge of the actual thickness and to use the effective thickness as a correction factor or apply a correction factor to the nominal thickness. Another way will be to find another method to measure the channel thickness.

\section{References}

1. J. Granger, J. Dodd, D. Leclerc, and N. Midoux, (1986)

2. K.-G. Wahlund and J. C. Giddings, Society 1332 (1987)

3. J. C. Giddings, Sep. Sci. 1, 123 (1966)

4. J. C. Giddings, 50, 667 (1973)

5. J. Gigault and V. A. Hackley, Anal. Bioanal. Chem. 405, 6251 (2013)

6. M. Martin, J. Chromatogr. A 831, 73 (1999)

7. Y. Mori, K. Kimura, and M. Tanigaki, Anal. Chem. 62, 2668 (1990)

8. E. Bolea, J. Jiménez-Lamana, F. Laborda, and J. R. Castillo, Anal. Bioanal. Chem. 401, 2723 (2011)

9. M. Correia and K. Loeschner, Anal. Bioanal. Chem. 410, 5603 (2018)

10. S. Schachermeyer, J. Ashby, M. Kwon, and W. Zhong, J. Chromatogr. A 1264, 72 (2012)

11. K. Hungerbühler, J. Ebert, A. Ulrich, B. Nowack, C. Adlhart, N. Bendixen, H. Hagendorfer, S. Losert, M. Lattuada, and A. Al-Kattan, J. Anal. At. Spectrom. 27, 1120 (2012)

12. S. Losert, N. Bendixen, K. Hungerbühler, and A. Ulrich, 1, 155 (2013)

13. H. Kato, A. Nakamura, H. Banno, and M. Shimizu, Colloids Surfaces A Physicochem. Eng. Asp. 538, $678(2018)$

14. K. G. Wahlund, J. Chromatogr. A 1287, 97 (2013)

15. M. E. Schimpf and Q. Du, Anal. Chem. 74, 2478 (2002)

16. H. Dou, E. C. Jung, and S. Lee, J. Chromatogr. A 1393, 115 (2015) 